\title{
Diseño estratégico para la innovación social y la sostenibilidad
}

\section{Strategic design for social innovation and sustainability}

\author{
Carlo Franzato, Universidade do Vale do Rio dos Sinos (Unisinos) \\ cfranzato@unisinos.br
}

Resumen

El discurso del diseño sostenible ha expresado la importancia de procesos proyectuales cada vez más estratégicos para su efectividad. La progresiva comprensión de la insostenibilidad de nuestros modelos de desarrollo lleva a que los expertos busquen innovaciones sociales sistémicas. Para el desarrollo de innovaciones de ese tipo son necesarios aportes metodológicos del diseño estratégico. Este artículo tiene como objetivo discutir esa problemática, que vincula estrechamente innovación social y sostenibilidad, e identificar las principales contribuciones del diseño estratégico. Para ello, realiza una revisión de los conceptos y de las relaciones entre el diseño y la sostenibilidad, y profundiza la labor de la DESIS, red de laboratorios de diseño para la innovación social y la sostenibilidad. En este recorrido, se identifican los principales aportes del diseño estratégico para la consecución de la sostenibilidad, finalmente discutidas a la luz de las teorías de la complejidad que inspiran esta vertiente metodológica. Además, el artículo elabora el concepto de 'sostenibilidad proyectual', sugiriendo que la sostenibilidad pueda considerarse constitutiva del proceso proyectual y no sólo su fin último.

Palabras clave: Diseño sostenible, diseño estratégico, sostenibilidad, sostenibilidad proyectual, complejidad.

\begin{abstract}
The discourse of sustainable design has shown the importance of increasingly strategic design processes for its effectiveness. The progressive understanding of our development models unsustainability leads specialists to look for systemic social innovations. In order to elaborate innovations of this type, methodological contributions of strategic design are necessary. This article aims to discuss this issue, which closely links social innovation and sustainability, and to identify the main contributions of strategic design. To this end, it operates a review of concepts and relationships between design and sustainability, and deepens the work of DESIS, a network of design laboratories for social innovation and sustainability. In this direction, the main contributions of strategic design to the search for sustainability are identified, being finally discussed from the perspective of complexity. In addition, the article elaborates on the concept of 'design sustainability', suggesting that sustainability can be considered a constituent of the design process and not just its end.
\end{abstract}

Keywords: Sustainable design, strategic design, sustainability, design sustainability, complexity. 


\section{Diseño y sostenibilidad}

Este artículo se centra en la sostenibilidad, entendida tanto como un desafío que los procesos de innovación social desarrollados por el diseño necesariamente deben enfrentar, cuanto como una oportunidad para el perfeccionamiento metodológico del mismo diseño.

La sostenibilidad es propia de los procesos de transformación del mundo que, al mismo tiempo que les posibilitan a las generaciones actuales el alcance de sus objetivos, garantizan a las generaciones futuras las mismas oportunidades. Según la Declaración de Río, elaborada en los trabajos de la Conferencia de las Naciones Unidas sobre el Medio Ambiente y el Desarrollo, que tuvo lugar en la ciudad carioca de 3 a 14 de junio de 1992, "el derecho al desarrollo debe ejercerse en forma tal que responda equitativamente a las necesidades de desarrollo y ambientales de las generaciones presentes y futuras" (CNUMAD, 1992, sin páginas). Entonces, en el contexto de la sostenibilidad, se hace indispensable evaluar si nuestras prácticas de desarrollo cumplen o sobrepasan la medida de la solidaridad entre las generaciones.

Así como el concepto de sostenibilidad, también el de diseño está relacionado con la transformación del mundo y tensado hacia el futuro (FRANZATO et al., 2015; FRANZATO, 2017). De esa manera, el diseño y la sostenibilidad establecen una relación en el horizonte del futuro. Sin embargo, los futuros del diseño y de la sostenibilidad no son exactamente los mismos. Mientras que el futuro del diseño es transformar el mundo, el futuro de la sostenibilidad es el que se deriva de esta transformación: la sostenibilidad es el futuro del futuro del diseño.

Pincipalmente desde la década del setenta, diversos especialistas del diseño, de la arquitectura, de las ingenierías y de otras áreas han aceptado el reto de la sostenibilidad. Sus actividades vienen centrándose especialmente en la dimensión ambiental, buscando desarrollar productos cuyos procesos de producción y consumo estén optimizados para causar el menor impacto en el medio ambiente. Sin embargo, a pesar de los buenos resultados obtenidos en las últimas tres décadas, ante la continuidad de la crisis ecológica, varios de estos expertos están empezando a constatar que el desarrollo de productos apenas más sostenibles que aquellos hasta entonces disponibles, no resuelve el problema de la insostenibilidad del desarrollo (véase, entre otros, MANZINI, VEZZOLI, 2003; CRUL, DIEHL, 2006; VEZZOLI et al., 2014). Como ellos sugieren, se vuelve cada vez más evidente la urgencia de buscar la sostenibilidad de manera más sistémica y a largo plazo. Ceschin y Gaziulusoy analizan esta evolución del diseño hacia la sostenibilidad, evidenciando cómo el método debe volverse cada vez más estratégico para responder al desafío en análisis (2016, p. 141).

Para presentar esos cambios en el área del diseño, la siguiente sección describe la propuesta de la red DESIS (Design for Social Innovation and Sustainability). Se trata de una red internacional de laboratorios de investigación y proyecto que se distribuyen en todos los continentes. En América Latina, DESIS cuenta con laboratorios incubados en las instituciones brasileñas UEMG, UFPB, UFPR, UFRJ, UFSC y UNISINOS, en la colombiana Universidad del Norte y en la chilena DUOC UC (DESIS, 2016). 


\section{Diseño, sostenibilidad e innovación social: la propuesta de la red DESIS}

La red DESIS parte de la constatación que es urgente un cambio de los modelos de desarrollo actuales, frente a su evidente insostenibilidad. Según Manzini, fundador y actual presidente de DESIS, el camino hacia la sostenibilidad requiere una "discontinuidad sistémica" que modifique la vigente idea de desarrollo y de bienestar (2008, p. 19, 25-28, 61). Demanda el paso de esta sociedad, que asocia su bienestar al continuo crecimiento de la capacidad de producción y consumo, a una sociedad que aprenda a desarrollarse "simultáneamente mejorando la calidad de todo el entorno social y físico" (2008, p. 19, traducción del autor). Así pues, la innovación social y la sostenibilidad convergen y se convierten en objetivos combinados del diseño. De acuerdo con la definición de la Young Foundation, llamamos de innovación social a la elaboración de nuevas ideas de productos, servicios y modelos que "satisfagan las necesidades sociales y simultáneamente creen nuevas relaciones o colaboraciones sociales. En otras palabras, son innovaciones buenas para la sociedad y que mejoran la capacidad de la sociedad de actuar" (MURRAY, CAULIER-GRICE, MULGAN, 2010, p. 3, traducción del autor).

Desarrollo y bienestar deben ser comprendidos de manera articulada, y buscados en la complejidad ecosistémica en la que vivimos. Esta relación es crucial en la propuesta de la red DESIS, porque ella es el principio estratégico que despliega el método proyectual practicado por sus miembros. La red DESIS busca crear las condiciones para que el cambio prospectado "pueda suceder no como una necesidad, sino como una elección" (MANZINI, 2008, p. 39, traducción del autor). El cambio no es impuesto y no debería pesar sobre la sociedad, sino ser buscado por ella, porque está insertado en el horizonte de un futuro deseable.

En ese sentido, la red DESIS pasa a la elaboración de escenarios, tal como ocurre normalmente en los procesos de diseño estratégico. Se trata de "escenarios de bienestar sostenible" (MANZINI, 2003a) que permitan la discusión colectiva de los actuales modelos de desarrollo, así como la proposición y evaluación de alternativas sostenibles, entonces presuponiendo la convergencia de los diversos actores involucrados en la consecución de metas comunes. La red elabora tales escenarios especialmente a través de dos procesos complementarios. El primero consiste en la recopilación, el estudio e intercambio de innovaciones emergentes en los diversos ámbitos de actividad de los miembros de la red, lo que puede constituir una base referencial para la elaboración de escenarios (MERONI, 2007). Se trata de prácticas sostenibles ya consolidadas en un contexto determinado, o simplemente prototípicas, a menudo desarrolladas sin la contribución de diseñadores profesionales y que abordan las cuestiones sociales con originalidad. Aisladas, las innovaciones recogidas no ilustran los escenarios, pero se ofrecen como indicios para su elaboración. Por medio de su articulación e interpretación, es posible vislumbrar posibilidades de futuros alternativos para la sociedad y oportunidades para el proyecto. El segundo proceso es la organización de talleres cocreativos "para concebir, visualizar y desarrollar escenarios y soluciones sostenibles, en el marco de los procesos amplios y articulados de coplanificación que caracterizan la innovación de los sistemas sociotécnicos contemporáneos" (MANZINI, 2003b, p. 233, traducción del autor). Según Manzini, para organizar tales talleres, el diseñador puede acceder al bagaje del diseño estratégico, considerando sus posibilidades procesuales e instrumentales para organizar una pluridiversidad de actores de la innovación social, en la coordinación de reuniones de cocreación y en la elaboración de escenarios (MANZINI, 2003c). 
En estos talleres, el diseñador se convierte en "un operador que puede facilitar la construcción de una visión compartida, a través de la creación y de la proposición" de esos escenarios y de soluciones sostenibles (MANZINI, 2003b, p. 233, traducción del autor).

La innovación es inmanente a los procesos de diseño de la red DESIS, potenciada siempre que sus miembros y, especialmente, los actores que actúan en las diversas organizaciones que se relacionan directa o indirectamente con la red - como agencias de diseño, comunidades o colectivos creativos, ONGs, empresas de impacto social, instituciones, administraciones públicas, entre otros - se apropien de los resultados de los procesos proyectuales y los implementen. Es por esa principal razón que la red busca extrapolar el ámbito académico y profesional del diseño, promoviendo la inclusión de esos diversos actores en sus talleres.

La red también puede contar con, por lo menos, dos procesos más, estrictamente relacionados con la innovación. A través de la organización de publicaciones (MERONI, 2007, JÉGOU, MANZINI, 2008), exposiciones (MANZINI, JÉGOU, 2003) y eventos (DESIS, 2015), la red elabora un proceso de construcción y "amplificación" (PENIN, KOBORI, FORLANO, 2012) del sentido de las proposiciones, a manera de aumentar su resonancia cultural. El segundo proceso busca la "diseminación" (FREIRE, DEL GAUDIO, FRANZATO, 2017) de esas proposiciones en contextos diferentes de aquellos que las originaron, por medio de oportunas apropiaciones y adaptaciones. Una buena parte de los esfuerzos de la red se centra en la concepción de ideas de nuevos sistemas producto-servicios, aunque exista otra parte, también significativa, centrada en el desarrollo de estrategias para la difusión de estos sistemas.

En ese sentido, no parece que la red DESIS esté interesada en soluciones que admitan una innovación disruptiva y radical, lo que representaría una excepción en el diseño contemporáneo. El radicalismo que puede ser atribuído a su propuesta está en los escenarios de bienestar sostenible que ella prospecta. Pretendiendo cambiar sensiblemente nuestra noción de estar en el mundo y de estar con los demás, la red vislumbra una innovación que, antes de ser social, podría ser considerada cultural (FRANZATO et al., 2015). Es social, pues, recuperando la definición de Murray, Caulier-Grice y Mulgan (2010, p. 3), pretende satisfacer las necesidades sociales, al mismo tiempo que teje relaciones entre los actores involucrados en el proceso de innovación y todos los ciudadanos. Siendo así, les permite acceder al proceso y fomentar su colaboración, hasta el punto que el diseñador pasa a actuar en un segundo plano, como un operador que sólo facilita la expresión de la creatividad difusa en la sociedad (MANZINI, 2017a). Primeramente, sin embargo, es cultural, porque su objetivo principal, más que la satisfacción de necesidades sociales, es el de imaginar nuevas propuestas para la convivencia de los seres humanos que habitan el planeta tierra. Es gracias a esta 'innovación cultural y social' que podrá haber una innovación sistémica, que podrá ocurrir una transición hacia otra sociedad, rumbo a futuros alternativos. 


\section{Los aportes del diseño estratégico}

La red DESIS recurre al diseño estratégico para la organización de sus talleres cocreativos y la elaboración de los escenarios de bienestar sostenible. En el trabajo de la red, sin embargo, el diseño estratégico tiene también la atribución de articular a los diversos actores implicados en los procesos proyectuales, es decir, de formar esas coaliciones (MANZINI, 2017) o redes proyectuales (MANZINI, 2008, p. 83-98) que promueven la innovación y por las que la innovación se disemina. Se trata de formas de organización que elaboran el proceso de diseño, al mismo tiempo en que son elaboradas por ese proceso. De hecho, Manzini afirma que "ellas mismas son un resultado de diseño: una actividad propia de la disciplina de diseño estratégico, que busca identificar un grupo adecuado de personas interesadas y construir valores comunes e intereses convergentes con ellos" (2015, p. 64, traducción del autor).

El diseño estratégico frecuentemente está relacionado al desempeño del diseño en los niveles estratégicos de las empresas (MAGALHÃES, 1997; MORALES, 2004; DI LUCCHIO, 2005; CAUTELA, ZURLO, 2006; LEIRO, 2006; FRANZATO, 2010; ZURLO, 2010; COUTINHO, PENHA, 2015), pero no debe resultar sorprendente que sea considerado más allá del ámbito empresarial, como ocurre en el trabajo de Manzini o de varios otros autores (MAURI, 1996; MERONI, 2008; CESCHIN Y GAZIULUSOY, 2016; FREIRE, DEL GAUDIO, FRANZATO, 2017; FRANZATO, 2017). De hecho, el proceso de diseño estratégico se relaciona con el proceso de organización, de cualquier tipo de organización del contexto sociotécnico y no sólo de las empresas. Por esta razón, la red DESIS recurre precisamente al diseño estratégico para organizar a los actores que participan en el proceso proyectual en coaliciones cohesionadas o en redes más sueltas y flexibles (MANZINI, 2015).

Esta posibilidad de organizar redes de proyecto constituye una importante competencia del diseño estratégico en un contexto sociotécnico en el que las tecnologías de la información se propagan sin límites aparentes, promoviendo la comunicación y la interacción de múltiples actores situados en las más diversas combinaciones espaciotemporales. De acuerdo con Manuel Castells:

La morfología de red parece estar bien adaptada para una complejidad de interacción creciente y para pautas de desarrollo impredecibles que surgen del poder creativo de esa interacción. Esta configuración topológica, la red, ahora puede materializarse en todo tipo de procesos y organizaciones mediante tecnologías de la información de reciente disposición. Sin ellas, sería demasiado engorroso poner en práctica la lógica de interconexión. No obstante, ésta es necesaria para estructurar lo no estructurado mientras se preserva su flexibilidad, ya que lo no estructurado es la fuerza impulsora de la innovación en la actividad humana (p. 88, 2005).

En esta fuerza creadora expresada por la complejidad de las interacciones sociales, reside un gran potencial para los procesos de diseño e innovación. Para servirse de él, sin embargo, es necesario hacer frente a la imprevisibilidad que es inherente a la complejidad y, por lo tanto, aceptar la pérdida de un supuesto control sobre los procesos. En 1996, emblemáticamente el mismo año en que Castells publicaba La Sociedad Red, obra de la cual se extrae el párrafo anterior, Francesco Mauri publicaba Proyectar proyectando estrategia (traducción del autor), seminal para el diseño estratégico, texto en el que anunciaba la necesidad de la renuncia "a la predeterminación y planificación proyectual, confrontando el proyecto con la posibilidad de 
enfrentar y gestionar el cambio, promover continuamente la innovación, operar estratégicamente" (1996, p. XI, traducción del autor).

En esa obra:

El proyecto es repensado como un saber creativo que se expresa en una dimensión del actuar relacional, no es concebido como un acto cumplido que precede a la ejecución, sino como un actuar susceptible de continuos cambios: el gerundio reiterativo [proyectando, que reitera el infinitivo proyectar, nota del autor] resalta el aspecto de actuar proyectual en el devenir, no programable y ni demarcable, capaz de auto-organización (MAURI, 1996, p. XI, traducción del autor).

Mauri desarrolla la propuesta del diseño estratégico a partir de las elaboraciones de autores del pensamiento complejo, como Edgar Morin (1977, 1985) o Jean-Louis Le Moigne (1985), que, a menudo, se interrogan sobre el proceso de proyectación, relacionándolo con el proceso de organización. Le Moigne llega a afirmar: "proyectar es organizar" (1985, p. 70).

Sobre la base del pensamiento complejo, se encuentra la noción de sistema abierto, es decir, utilizando las palabras de Morin, un sistema que organiza "su clausura (es decir su autonomía) en y por su apertura" (1995, p. 42). Morin sigue señalando dos importantes implicaciones que derivan de la noción de sistema abierto. La primera es que las leyes de organización de esos sistemas "no son de equilibrio, sino de desequilibrio, retomado o compensado, de dinamismo estabilizado" (MORIN, 1995, p. 42). Los sistemas abiertos están constituidos por una red dinámica de relaciones pluridiversificadas entre sus elementos, y se organizan incesantemente por una red dinámica de procesos. De esta forma, logran mantener cierta estabilidad sistémica, sin jamás alcanzar el equilibrio, ya que el equilibrio implicaría su fenecimiento. La segunda implicación es la necesidad de comprender los sistemas abiertos desde una perspectiva ecosistémica: "la inteligibilidad del sistema debe encontrarse no solamente en el sistema mismo, sino también en su relación con el ambiente, y esa relación no es una simple dependencia, sino que es constitutiva del sistema" (MORIN, 1995, p. 42).

En ese sentido, la propuesta de Mauri se desdobla desde una perspectiva ecosistémica, no concentrándose específicamente en el desarrollo de productos o sistemas producto-servicio más eficientes, sino en un proyecto de estrategias que promuevan el desarrollo de relaciones ecosistémicas y así aumenten las capacidades de renovación y resiliencia del ecosistema. Con la expresión "proyecto de la estrategia", Mauri se refiere a "un actuar proyectual transdisciplinario, capaz de conectar diferentes puntos de vista y de tejer competencias y funciones interdependientes", pues, continúa el autor, "múltiples contribuciones compiten para formarla in itinere" (1996, p. 38, traducción del autor). Con 'diseño estratégico' Mauri se refiere a la contribución específica del diseño, en el enmarañado transdisciplinario del proyecto de la estrategia.

En la elección del término 'estrategia' como palabra clave del discurso proyectual, Mauri (1996, p. 27) recurre nuevamente a Morin, citando un fragmento emblemático del filósofo francés:

La acción es estrategia. La palabra estrategia no designa a un programa predeterminado que baste aplicar ne varietur en el tiempo. La estrategia permite, a partir de una decisión inicial, imaginar un cierto número de escenarios para la acción, escenarios que podrán ser; modificados según las informaciones que nos lleguen en el curso de la acción y según los elementos aleatorios que sobrevendrán y perturbarán la acción (MORIN, 1995, 113). 
En esas palabras, vuelve el concepto de escenario ya encontrado en el corazón de los talleres cocreativos que la red DESIS organiza y que sirven de base para el diseño estratégico. La elaboración de escenarios no es una actividad accesoria del diseño estratégico, sino que, por el contrario, es su constituyente (MANZINI, JÉGOU, 2003; MORALES, 2004; HARTMANN, FRANZATO, 2012; COUTINHO, PENHA, 2015). Por medio de los escenarios, el diseño estratégico logra agregar los diversos actores que participan del trabajo colectivo de imaginación de otros futuros, futuros posibles y, posiblemente alternativos, que hospedan el proyecto.

\section{Conclusiones: para una sostenibilidad proyectual}

Desde Proyectar proyectando estrategia, Mauri afirmaba la necesidad de "volver a pensar y a discutir radicalmente el sentido de la proyectualidad, anclándolo en la búsqueda de posibles direcciones de coevolución de los sistemas humanos y naturales", a la luz de la "urgencia improrrogable de encontrar perspectivas de sostenibilidad" (1996, p. 48, traducción del autor).

Tras el análisis del trabajo de la red DESIS y de recurrir a los desarrollos teóricos de Mauri, en la última sección se presentaron algunas de las aportaciones del diseño estratégico para que el diseño y todos los procesos proyectuales puedan contemplar la sostenibilidad, así como la innovación cultural y social. Entre ellas, se encuentra la creación de coaliciones y redes de proyecto, bien como la elaboración de escenarios. La red DESIS se enfrenta a la insostenibilidad de los modelos de desarrollo actuales y, por lo tanto, entiende que es insuficiente buscar soluciones que, aunque innovadoras, sean puntuales y apenas más sostenibles. Propone, entonces, que el diseño colabore en un proceso de innovación social más amplio, operado por la constitución de redes de proyecto que nos permitan imaginar y realizar nuevos escenarios de bienestar sostenible, o sea, escenarios en que el bienestar de las personas esté relacionado con formas plenamente sostenibles de estar en el mundo y con los demás.

A partir de la comprensión de que la búsqueda por la sostenibilidad es un reto colectivo, de la adopción del pensamiento complejo y de una perspectiva ecosistémica, la contribución conceptual más marcante es de una nueva comprensión del diseño como 'actuar organizacional'. En esta locución, inspirada en las sugerencias de Mauri, Le Moigne y Morin, están incluidos los intereses del diseño en la práctica de relaciones y en la elaboración de procesos, especialmente de los procesos estratégicos.

El diseño estratégico, al emerger de las dinámicas proyectuales de los sistemas organizativos, actúa en esas dinámicas para fortalecerlas. Por las complejas redes de relaciones que articulan los sistemas, ocurre una intensa actividad procesal: procesos que desencadenan procesos que, a su vez, desencadenan otros procesos hasta volver a reforzar los primeros, cerrando el ciclo. En ese sentido, como Mauri resalta, el diseño no puede ser considerado como un acto cumplido que precede a sus efectos transformadores: productos, servicios, modelos, etc. Debe ser considerado como un actuar situado en el hic et hunc de nuestras prácticas cotidianas, capaz de transformaciones, al mismo tiempo en que él mismo sufre continuas transformaciones.

Las estrategias que se despliegan en este enmarañado de relaciones y procesos buscan justamente constituir esa trama organizacional, y son innovadoras porque contribuyen por la 
eficiencia, resiliencia y resistencia del sistema, en su relación mutua con el ecosistema, hasta alcanzar la sostenibilidad. Así, las transformaciones expresadas en los procesos de diseño estratégico deben ser evaluadas por su capacidad de mantener y crear condiciones favorables, para que exista la posibilidad de nuevas transformaciones en el futuro. Es en ese sentido que, siguiendo las propuestas de Mauri o Manzini, es necesario que el diseño estratégico se enfrente al reto de la sostenibilidad. La gran complejidad y dificultad de ese desafío demanda a los diseñadores a la adopción de perspectivas y de modalidad de acción que les permitan operar de forma sistémica y en tiempo real, considerando la red de relaciones y procesos organizacionales.

En esa comprensión - anclada en los conceptos de organización, sistema, ecosistema - diseño, estrategia, innovación y sostenibilidad resultan transversal y necesariamente articulados. Su entrelazado e indisociabilidad pueden ser analizados tanto de manera diacrónica, como sincrónica. En los discursos del diseño, de la estrategia, de la innovación y de la sostenibilidad, se privilegia un tipo de lectura diacrónica que dispone la secuencia procesal en la línea del tiempo, para evaluar prospectivamente su encadenamiento causal. De esa manera, la sostenibilidad es vista como un resultado procesal, un alcance resultante de los procesos de diseño, elaboración estratégica e innovación. En el discurso del diseño sostenible, la sostenibilidad es vista específicamente como un fin último (MAURI, 1996; MANZINI, VEZZOLI, 2003; JÉGOU, MANZINI, 2008; MANZINI, 2008; VEZZOLI et al., 2014; CESCHIN, GAZIULUSOY, 2016; CRUL, DIEHL, 2017).

En estas conclusiones, se propone operar también un tipo de lectura sincrónica de ese entretejido que considere los procesos organizacionales sistémicamente y sin desplegarlos en la línea del tiempo por la relación de causa y efecto. Es importante resaltar que no se sugiere evaluar los procesos en un momento dado, pero sí el sistema de procesos en los diversos tiempos o independientemente del tiempo. Por lo tanto, se cuestiona si la sostenibilidad es un mero resultado o el fin de los procesos y de su organización sistémica, o si es su constituyente. En esta lectura, la sostenibilidad de los sistemas y de su ecosistema de referencia, al identificar los primeros sin poder separarlos de los segundos, no podría ser considerada únicamente como un fin desplazado en el futuro, sino más bien como una condición para su existencia. Por lo tanto, si hay organización, si hay entrelazamiento de procesos, si existen cada uno de los procesos, incluso el diseño, es porque se establece una condición que sostiene su existencia, es decir, hay sostenibilidad. Es evidente que la sostenibilidad de los sistemas y del ecosistema puede y debe ser también un fin último de la organización, en una dinámica de "auto-organización" (MORIN, 1995, CAPRA, 1996). Como fin, la sostenibilidad es un atributo del diseño, bien expresado en los escritos de diseño sostenible o diseño para la sostenibilidad, entre otros, aún así cualquier tipo de proceso de diseño debería anhelar su búsqueda y alcance. Como constituyente originario del proceso proyectual, la sostenibilidad se vuelve intrínseca al diseño. Esta inversión conduce a la identificación de una 'sostenibilidad proyectual' como condición sistémica para que exista la posibilidad de procesos de diseño. 


\section{Referencias}

CAPRA, F. A teia da vida. São Paulo: Cultrix, 1996.

CASTELLS, M. La sociedad red. Buenos Aires: Siglo XXI, 2005.

CAUTELA, C.; ZURLO, F. Relazioni produttive. Design e strategia nell'impresa contemporanea. Roma: Aracne, 2006

CESCHIN, F.; GAZIULUSOY, I. Evolution of design for sustainability: From product design to design for system innovations and transitions. Design Studies, 47, p. 118-163, 2016. Disponível em: https://www.sciencedirect.com/science/article/pii/S0142694X16300631, acesso em 26 de agosto de 2018.

CNUMAD. Declaración de Río sobre el Medio Ambiente y el Desarrollo. Rio de Janeiro: CNUMAD, $1992 . \quad$ Disponível em: http://www.un.org/spanish/esa/sustdev/documents/declaracionrio.htm, acesso em $26 \mathrm{de}$ agosto de 2018.

COUTINHO, A.; PENHA, A. Design estratégico a partir do futuro. Harvard Business Review Brasil, setembro, 2015. Disponível em: http://hbrbr.uol.com.br/design-estrategicopartir-do-futuro/, acesso em 26 de agosto de 2018.

CRUL, M.; DIEHL, J. C. 2017. Design for sustainability: a practical approach for developing economies. Paris-Delft: UNEP-TUDelft. Disponível em: http://wedocs.unep.org/handle/20.500.11822/8720, acesso em 26 de agosto de 2018.

DESIS. DESIS network showcase. 2015. Disponível em: http://www.desis-showcase.org/, acesso em 26 de agosto de 2018.

DESIS. $\quad$ Labs. $2016 . \quad$ Disponível em: http://www.desisnetwork.org/labs/?filter category=south-america, acesso em 26 de agosto de 2018.

DI LUCCHIO, L. Il design delle strategie. Un modello interpretativo della relazione tra design e impresa. Roma: Gangemi, 2005.

FRANZATO, C. et al. Inovação Cultural e Social: design estratégico e ecossistemas criativos. In: FREIRE, K. (org.). Design Estratégico para a Inovação Cultural e Social. São Paulo: Kazuá, p. 157-182, 2015.

FRANZATO, C. O design estratégico no diálogo entre cultura de projeto e cultura de empresa. Strategic Design Research Journal, v. 3, n. 3, p. 89-96, 2010. Disponível em: http://revistas.unisinos.br/index.php/sdrj/article/view/4791, acesso em 26 de agosto de 2018.

FRANZATO, C. Redes de projeto: formas de organização do design contemporâneo em direção à sustentabilidade. In: OLIVEIRA, A. J.; FRANZATO, C.; DEL GAUDIO, C. (orgs.). Ecovisões projetuais: pesquisas em design e sustentabilidade no Brasil. São Paulo: Blucher, 2017, p. 99-110. https://openaccess.blucher.com.br/article-details/09-20544, acesso em 26 de agosto de 2018.

FREIRE, K.; DEL GAUDIO, C. FRANZATO, C. Design-driven strategies for creative social innovation ecosystems. International Journal of Knowledge Engineering and Management, v. 6, n. 16, p. 46-69, 2017. Disponível em: http://incubadora.periodicos.ufsc.br/index.php/IJKEM/article/view/4709, acesso em $26 \mathrm{de}$ agosto de 2018.

HARTMANN, P.; FRANZATO, C. Design de cenários: uma tecnologia para promover o compartilhamento de conhecimentos em redes de projeto. Revista D: Design, Educação, Sociedade e Sustentabilidade, v. 4, n. 4, p. 155-168, 2012. Disponível em: http://seer.uniritter.edu.br/index.php/revistadesign/article/view/719, acesso em 26 de agosto de 2018. 
JÉGOU, F.; MANZINI, E. Collaborative services: social innovation and design for sustainability. Milano: POLI.design, 2008. Disponível em: http://www.strategicdesignscenarios.net/collaborative-services-book/, acesso em 26 de agosto de 2018.

LE MOIGNE, J. L. Progettazione della complessità e complessità della progettazione. In: BOCCHI, G.; CERUTI, M. (orgs.). La sfida della complessità. Milano: Feltrinelli, p. 60-78, 1985.

LEIRO, R. J. Diseño: Estrategia y gestión. Buenos Aires: Infinito, 2006.

MAGALHÃES, C. F. Design Estratégico. Rio de Janeiro: CNI/Senai-Cetiqt, 1997.

MANZINI, E. Design para a inovação social e sustentabilidade. Comunidades criativas, organizações colaborativas e novas redes projetuais. Rio de Janeiro: e-papers, 2008.

MANZINI, E. Design workshop: a methodology for generating new ideas, and catalysing existing ones. In MANZINI, E.; JÉGOU, F. (orgs.) Sustainable everyday. Scenarios of urban life. Milano: Ambiente, p. 242-243, 2003c. Disponível em: http://www.strategicdesignscenarios.net/wp-content/uploads/2012/05/SUSTAINABLEEVERYDAY -Scenarios-of-urban-life.pdf, acesso em 26 de agosto de 2018.

MANZINI, E. Design, when everybody designs. An introduction to design for social innovation. Cambridge, US: MIT Press, 2015.

MANZINI, E. Designing coalitions: Design for social forms in a fluid world. Strategic Design Research Journal, v. 10, n. 2, p. 187-193, 2017. Disponível em: http://revistas.unisinos.br/index.php/sdrj/article/view/sdrj.2017.102.12, acesso em 26 de agosto de 2018.

MANZINI, E. Strategic design for sustainability: instruments for radically oriented innovation. In MANZINI, E.; JÉGOU, F. (orgs.) Sustainable everyday. Scenarios of urban life. Milano: Ambiente, p. 231-234, 2003b. Disponível em: http://www.strategicdesignscenarios.net/wp-content/uploads/2012/05/SUSTAINABLEEVERYDAY_-Scenarios-of-urban-life.pdf, acesso em 26 de agosto de 2018.

MANZINI, E.; JÉGOU, F. Sustainable everyday. Scenarios of urban life. Milano: Ambiente, 2003. Disponível em: http://www.strategicdesignscenarios.net/wpcontent/uploads/2012/05/SUSTAINABLE-EVERYDAY_-Scenarios-of-urban-life.pdf, acesso em 26 de agosto de 2018.

MANZINI, E.; VEZZOLI, C. A strategic design approach to develop sustainable product service systems: examples taken from the "environmentally friendly innovation" Italian prize. Journal of Cleaner Production, v. 11, n. 8, p. 851-857, 2003.

MANZINI, M. Scenarios of Sustainable Wellbeing. Design Philosophy Papers, v. 1, n. 1, p. 5-21, 2003a.

MAURI, F. Progettare progettando strategia: il design del sistema prodotto. Milano: Dunod, 1996.

MERONI, A. Creative communities. People inventing sustainable ways of living. Milano: POLI.design, 2007. Disponível em: http://www.strategicdesignscenarios.net/creativecommunities-book/, acesso em 26 de agosto de 2018.

MERONI, A. Strategic design: where are we now? Reflection around the foundations of a recent discipline. Strategic Design Research Journal, v. 1, n. 1, p. 31-38, 2008. Disponível em: http://revistas.unisinos.br/index.php/sdrj/article/view/5567, acesso em 26 de agosto de 2018.

MORALES, L. R. Diseño: estrategia y táctica. Ciudad de México: Siglo XXI, 2004.

MORIN, E. Introducción al pensamiento complejo. Barcelona: Gedisa, 1995. 
MORIN, E. La méthode. 1 La nature de la nature. Paris: Editions du Seuil, 1977.

MORIN, E. Le vie della complessità. In: BOCCHI, G.; CERUTI, M. (orgs.). La sfida della complessità. Milano: Feltrinelli, p. 25-36, 1985.

MURRAY, R.; CAULIER-GRICE, J.; MULGAN, G. The open book of social innovation. London: Young Foundation/NESTA, 2010. Disponível em: https://youngfoundation.org/publications/the-open-book-of-social-innovation/, acesso em 26 de agosto de 2018.

PENIN, L.; KOBORI, M.; FORLANO, L. Amplifying creative communities new york city. New York: Parsons DESIS lab, 2012. Disponível em: http://www.amplifyingcreativecommunities.org/AmplifyBook.pdf, acesso em 26 de agosto de 2018.

VEZZOLI, C.; KOHTALA, C.; SRINIVASAN, A.; DIEHL, J. C.; FUSAKUL, S. M.; XIN, L.; SATEESH, D.; Product-service system design for sustainability. Sheffield, UK: Greenleaf, 2014.

\section{Agradecimientos}

Este trabajo fue desarrollado con el apoyo de la Consejo Nacional de Desarrollo Científico y Tecnológico (CNPq) del Ministerio de Ciencia, Tecnología e Innovación de Brasil.

\section{Acerca del autor}

Carlo Franzato es diseñador y Doctor en Diseño por el Politecnico di Milano. Es profesor de Diseño Estratégico en la Universidade do Vale do Rio dos Sinos (UNISINOS). En la perspectiva de la complejidad y el alcance metodológico del diseño estratégico, su investigación tiene como tema central las redes de proyecto que se constituyen con la apertura del proceso de diseño para la pluridiversidad de relaciones de colaboración entre los actores de los ecosistemas creativos.

cfranzato@unisinos.br 